Severe or critical hypotension during post cardiac arrest care is associated with factors available on admission - a post hoc analysis of the TTH48 trial

Hästbacka, Johanna

2021-02

Hästbacka, J , Kirkegaard, H , Soreide , E, Taccone , F S , Rasmussen, B S , Storm , C , Kjaergaard, J , Laitio , T , Duez , C H V , Jeppesen, A N , Grejs , A M \& Skrifvars , M B 2021 , ' Severe or critical hypotension during post cardiac arrest care is associated with factors available on admission - a post hoc analysis of the TTH48 trial ' , Journal of Critical Care , vol. 61 , pp. 186-190 . https://doi.org/10.1016/j.jcrc.2020.10.026

http://hdl.handle.net/10138/339934

https://doi.org/10.1016/j.jcrc.2020.10.026

cc_by_nc_nd

acceptedVersion

Downloaded from Helda, University of Helsinki institutional repository.

This is an electronic reprint of the original article.

This reprint may differ from the original in pagination and typographic detail.

Please cite the original version. 


\title{
Severe or critical hypotension during post cardiac arrest care is associated with factors available on admission - a post hoc analysis of the TTH48 trial
}

\author{
Johanna Hästbacka ${ }^{1}$, Hans Kirkegaard ${ }^{2}$, Eldar Søreide ${ }^{3}$, Fabio Silvio Taccone ${ }^{4}$, Bodil Steen \\ Rasmussen $^{5}$, Christian Storm ${ }^{6}$, Jesper Kjaergaard 7 , Timo Laitio ${ }^{8}$, Christophe Henri Valdemar Duez $^{2}$, \\ Anni N Jeppesen², Anders M Grejs ${ }^{2}$, Markus B Skrifvars ${ }^{9}$
}

1. Division of Intensive Care medicine, Department of Anesthesiology, Intensive care and Pain medicine, University of Helsinki and Helsinki University Hospital, Helsinki, Finland

2. Research Center for Emergency Medicine and Department of Anesthesiology and Intensive Care Medicine, Aarhus University Hospital and Aarhus University, Aarhus, Denmark

3. Department of Anesthesiology and Intensive Care, Stavanger University Hospital, Stavanger, Norway, Department of Clinical Medicine, University of Bergen, Bergen, Norway

4. Department of Intensive Care, Erasme Hospital, Université Libre de Bruxelles (ULB), Brussels, Belgium

5. Department of Anesthesiology and Intensive Care Medicine, Aalborg University Hospital, and Clinical Institute, Aalborg University, Aalborg, Denmark

6. Department of Internal Medicine, Nephrology and Intensive Care, CharitéUniversitätsmedizin Berlin, Berlin, Germany

7. Department of Cardiology, Copenhagen University Hospital, Rigshospitalet, Copenhagen, Denmark

8. Division of Perioperative Services, Intensive Care Medicine and Pain Management, Turku University Hospital and University of Turku, Finland

9. Department of Emergency Care and Services, University of Helsinki and Helsinki University Hospital, Helsinki, Finland

Corresponding author: Dr Johanna Hästbacka

Address: Intensive Care Units, Helsinki University Hospital, PO Box 340,

Haartmaninkatu 4, 00290 Helsinki, Finland

E-mail: johanna.hastbacka@hus.fi 


\section{Abstract}

Purpose: We explored whether severe or critical hypotension can be predicted, based on patient and resuscitation characteristics in out-of-hospital cardiac arrest (OHCA) patients. We also explored the association of hypotension with mortality and neurological outcome.

Materials and methods: We conducted a post hoc analysis of the TTH48 study (NCT01689077), where 355 out-of-hospital cardiac arrest (OHCA) patients were randomized to targeted temperature management (TTM) treatment at $33^{\circ} \mathrm{C}$ for either 24 or 48 hours. We recorded hypotension, according to four severity categories [mild= MAP (mean arterial pressure) $>60 \mathrm{mmHg}$; moderate $=$ MAP $>60 \mathrm{mmHg}$ with full treatment; severe $=$ MAP $50-60 \mathrm{mmHg}$ despite full treatment; critical= MAP $<50 \mathrm{mmHg}$ for $>10$ minutes despite full treatment], within four days from admission. We used multivariable logistic regression analysis to test association of admission data with severe or critical hypotension.

Results: Diabetes mellitus (OR 3.715, 95\% Cl 1.180-11.692), longer ROSC delay (OR 1.064, $95 \% \mathrm{Cl}$ 1.022-1.108), admission MAP (OR 0.960, 95\% Cl 0.929-0.991) and non-shockable rhythm (OR 5.307, 95\% Cl 1.604-17.557) were associated with severe or critical hypotension. Severe or critical hypotension was associated with increased mortality and poor neurological outcome at 6 months.

Conclusions: Diabetes, non-shockable rhythm, longer delay to ROSC and lower admission MAP were predictors of severe or critical hypotension. Severe or critical hypotension was associated with poor outcome.

Keywords: Out-of-hospital cardiac arrest, Hemodynamics, Hypotension, Targeted Temperature Management, Predicting, Outcome 


\section{List of abbreviations}

BLS= Basic life support

$\mathrm{CA}=$ Cardiac arrest

$\mathrm{CHF}=$ Chronic heart failure

COPD $=$ Chronic obstructive pulmonary disease

$\mathrm{CPC}=$ Cerebral Performance category

$\mathrm{CPR}=$ cardio-pulmonary resuscitation

CV-SOFA = Cardio-vascular Sequential Organ Failure Assessment

$\mathrm{ICU}=$ Intensive Care Unit

$\mathrm{IQR}=$ Inter-quartile range

$\mathrm{MAP}=$ Mean arterial pressure

OHCA= Out-of-hospital cardiac arrest

$\mathrm{PCl}=$ Percutaneous coronary intervention

ROSC $=$ Return of spontaneous circulation

$\mathrm{SD}=$ standard deviation

TTM=Targeted temperature management 


\section{Background}

Targeted temperature management (TTM) after out-of-hospital cardiac arrest (OHCA) is an effective treatment option during post-cardiac arrest care, but it may be associated with side effects, such as severe hemodynamic instability. [1-3] Although most studies on TTM have excluded patients with cardiogenic shock [1-3], some initially stable patients experienced hypotension or hemodynamic instability [1, 3], required vasopressors [2] and presented arrhythmias [1] during TTM treatment. These are also the most common reasons for preterm rewarming [1-3] or for slightly raising the target temperature during the hypothermia phase.

In a recent randomized controlled trial comparing 24 and 48 hours duration of TTM, no difference was found in the neurological outcome or mortality between the treatment groups, but the patients assigned to receive 48 hours of TTM experienced more often mild hypotensive complications than the patients in the 24 hour treatment group. [3] Although the optimal hemodynamic targets for the post-OHCA treatment in the ICU are not well established, worse outcomes have been associated with lower mean arterial pressure levels in the early phase after arrest $[4,5]$, which makes avoiding hypotension a reasonable therapeutic strategy.

A better understanding of the risk factors responsible for developing severe or critical hypotension during TTM would facilitate recognizing patients who may need advanced hemodynamic monitoring. The purpose of this study was therefore to explore which factors, available on ICU admission, could predict the development of severe or critical hypotension during the first four days of post-cardiac arrest treatment. Also, we investigated whether duration of cooling would influence the occurrence of severe or critical hypotension during the post cardiac arrest care and how occurrence of hypotension would affect patients' outcomes. 


\section{Methods}

This is a post hoc sub-study of the TTH48 study (NCT01689077), including 355 OHCA patients. The study protocol and the results of the study are described in detail elsewhere $[1,6]$. Briefly, after informed consent of next of kin, unconscious (GCS $<8$ ) OHCA patients, aged 18-80, with a presumed cardiac origin of cardiac arrest were randomized to receive mild therapeutic hypothermia $\left(33 \pm 1^{\circ} \mathrm{C}\right)$ for either $24(\mathrm{~N}=179)$ or 48 hours $(\mathrm{N}=175)$. Exclusion criteria are reported in the study protocol and included persistent cardiogenic shock and systolic blood pressure $<80 \mathrm{mmHg}$ despite vasoactive treatment and/or aortic balloon pump intervention. Patients with acute neurological insults such as acute intra-cerebral bleeding or stroke were excluded as were patients needing acute coronary bypass surgery. The primary outcome of the trial was Cerebral Performance Category (CPC) score at six-months following the event, secondary outcome was 6month mortality. The ethical approval for the original study was obtained at each participating center.

Patient data used for this sub-study were retrieved from the electronic CRF database. Occurrence of hypotension was recorded daily. We focused on data from admission through the fourth day in the ICU care in this study. Hypotension was defined as in the TTH48 study protocol as "mild" if MAP (mean arterial pressure) was $>60 \mathrm{mmHg}$ with one vasopressor, "moderate" if MAP was $>60 \mathrm{mmHg}$ with full treatment (more than one vasopressor and/or inotropes) and "severe" when MAP was 50-60mmHg with full treatment. [3 (Supplement), 6] Hypotension was defined "critical" when MAP was $<50 \mathrm{mmHg}$ more than 10 minutes despite full treatment (more than one vasopressor and/or inotropes).

\section{Statistical analysis}


Frequencies of categorical variables are expressed as numbers and percentages and compared using Chi-square test or Fisher's exact test, when appropriate. Continuous variables are expressed as means with standard deviation $( \pm S D)$ when normally distributed and medians with interquartile ranges (IQR) when non-normally distributed. Non-normally distributed continuous variables were compared using the Wilcoxon Mann-Whitney U-test for two, and Kruskall-Wallis test for several groups. We used univariate logistic regression analysis to assess the associations of individual variables with outcome. These variables were chosen to the univariable analyses from data available on admission or soon after. They were included in the univariable analyses only if the potential association was perceived physiologically plausible or was based on literature. Redundancy was avoided by choosing the most robust variables if related (diabetes over glucose value or body mass index, etc). We then performed stepwise backward multivariable logistic regression analysis including the variables with $p<0.2$ in the univariate analyses. We created Kaplan-Meier curves for examining the differences in survival between groups. Statistical analyses were performed using SPSS statistics version 22.0 (IBM Corp, Armonk, NY, USA). Statistical significance was considered at the level of $p<0.05$.

\section{Results}

Three patients of the $\mathbf{3 5 5}$ originally randomised were not included in the analyses (two withdrew consent and one did not receive intervention). A total of 352 patients were included in this sub-study. One hundred and ninety-four (55.1\%) patients experienced hypotension of any severity. The severity of hypotension in all patients and according to length of cooling are presented in Table 1. 

Table 1. Incidence and severity of hypotension during the first four days after OHCA in the ICU

\begin{tabular}{|l|l|l|l|}
\hline & All, N=352 (\%) & $\begin{array}{l}\text { 24-hour TTM, } \\
\mathrm{N}=177(\%)\end{array}$ & $\begin{array}{l}\text { 48-hour TTM } \\
\mathrm{N}=175(\%)\end{array}$ \\
\hline $\begin{array}{l}\text { Hypotension } \\
\text { No hypotension }\end{array}$ & $158(44.9)$ & $91(51.4)$ & $67(38.3)$ \\
Mild & $113(32.1)$ & $54(30.5)$ & $59(33.7)$ \\
Moderate & $53(15.1)$ & $21(11.3)$ & $32(18.3)$ \\
Severe & $15(4.3)$ & $5(2.8)$ & $10(5.7)$ \\
Critical & $13(3.7)$ & $6(3.4)$ & $7(4.0)$ \\
\hline
\end{tabular}

$\mathrm{OHCA}=$ Out-of-hospital cardiac arrest; ICU= Intensive care unit; TTM= Targeted temperature management. The data are given as numbers and percentages.

Characteristics of patients, cardiac arrest (CA) and early treatment, according to the occurrence of severe or critical hypotension during the post CA care, are shown in Table 2. 
Table 2. Baseline and treatment characteristics according to occurrence of severe or critical hypotension

\begin{tabular}{|l|l|l|l|}
\hline & \multicolumn{2}{l}{ Severe or critical hypotension } & Yes \\
& \multicolumn{2}{l}{ No } & \\
\hline Median age, years (IQR) & $61(53-69)$ & $69(55-72)$ & 0.066 \\
\hline Gender male, N (\%) & $273(84.3)$ & $20(71.4)$ & 0.081 \\
\hline Chronic heart failure, N (\%) & $15(4.6)$ & $3(10.7)$ & 0.165 \\
\hline COPD, N (\%) & $22(6.8)$ & $2(7.1)$ & 1.00 \\
\hline $\begin{array}{l}\text { Previous myocardial infarction, } \\
\text { N (\%) }\end{array}$ & $48(14.9)$ & $6(21.4)$ & 0.364 \\
\hline Previous cardiac arrest, N (\%) & $3(0.9)$ & $0(0)$ & 1.000 \\
\hline Diabetes mellitus, N (\%) & $51(15.8)$ & $12(42.9)$ & $<0.001^{*}$ \\
\hline Bystander CPR, N (\%) & $273(84.3)$ & $20(71.4)$ & 0.08 \\
\hline Non-shockable rhythm, N (\%) & $33(10.2)$ & $7(25)$ & $0.018^{*}$ \\
\hline $\begin{array}{l}\text { Time to ROSC, min, median } \\
\text { (IQR) }\end{array}$ & $20(15-27)$ & $28.5(17-42.3)$ & $0.007^{*}$ \\
\hline $\begin{array}{l}\text { Mechanical chest compressions } \\
\text { device used, N (\%) }\end{array}$ & $80(25)$ & $10(37)$ & 0.171 \\
\hline $\begin{array}{l}\text { Immediate coronary } \\
\text { angiography, N (\%) }\end{array}$ & $272(84)$ & $19(67.9)$ & $0.031^{*}$ \\
\hline $\begin{array}{l}\text { Percutaneous coronary } \\
\text { intervention, N (\%) }\end{array}$ & $134(41.5)$ & $11(40.7)$ & 0.94 \\
\hline $\begin{array}{l}\text { Median MAP on ICU admission, } \\
\text { mmHg (IQR) }\end{array}$ & $79(67.3-92)$ & $69.5(59-83.8)$ & $0.025^{*}$ \\
\hline $\begin{array}{l}\text { Intervention group } \\
\text { 24h hypothermia, N (\%) } \\
\text { 48h hypothermia, N (\%) }\end{array}$ & $166(51.2)$ & $11(39.3)$ & 0.225 \\
\hline $\begin{array}{l}\text { Median lactate on ICU } \\
\text { admission, mmol/L (IQR) }\end{array}$ & $2.6(1.4-4.7)$ & $4.0(1.53-9.2)$ & 0.088 \\
\hline
\end{tabular}

$\mathrm{SD}=$ standard deviation; $\mathrm{COPD}=$ Chronic obstructive pulmonary disease; $\mathrm{CPR}=$ cardiopulmonary resuscitation; $\mathrm{ROSC}=$ Return of spontaneous circulation; $\mathrm{IQR}=$ inter-quartile range; $\mathrm{MAP}=\mathrm{Mean}$ arterial blood pressure; ICU = Intensive Care Unit; ${ }^{*}=$ statistically significant, $p<0.05$ 


\section{Factors predicting severe or critical hypotension}

Univariable and multivariable analysis of factors that were associated with increased risk for severe or critical hypotension on days 1-4 are shown in Table 3. Diabetes as a comorbidity was strongly predictive of severe or critical hypotension, increasing the risk up to four-fold compared to non-diabetics. Twelve (19\%) of the 63 patients with diabetes experienced severe or critical hypotension, whereas $16(5.6 \%)$ of the 288 non-diabetic patients had severe or critical hypotensive complications $(p<0.001)$. Other factors independently and significantly associated with the risk of severe or critical hypotension were non-shockable rhythm, ROSC delay and MAP on admission. 
Table 3. Patient and treatment-related factors present on admission or early ICU stay and their association with occurrence of a severe or critical hypotension during ICU days 1-4.

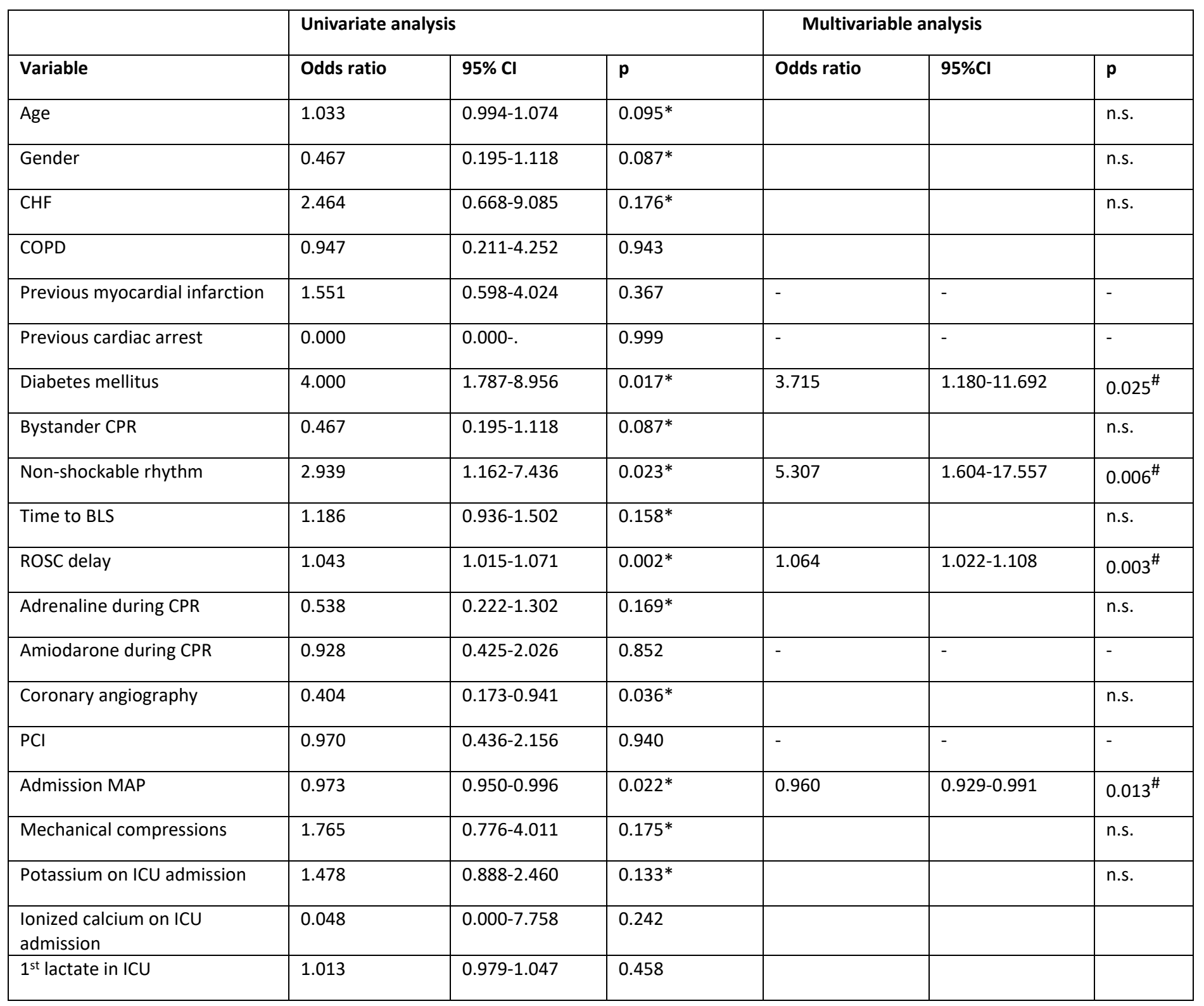

Variables marked with * achieved a significance level of $p<0.2$ on univariate analysis and were included in the stepwise backward logistic regression analysis with the exception of ICU admission glucose concentration which was not included due to its relationship with diabetes. The variables significantly ( $p<0.05$ was considered significant in the multivariate analysis) and independently associated with severe hypotension in the multivariate analysis are reported and marked with \#. $\mathrm{CHF}=$ Chronic heart failure (New York heart Association class 4); $\mathrm{COPD}=$ Chronic obstructive pulmonary disease; $\mathrm{CPR}=$ cardio-pulmonary resuscitation; $\mathrm{BLS}=$ Basic life support; $\mathrm{ROSC}=$ Return of 
spontaneous circulation; $\mathrm{PCl}=\mathrm{Percutaneous}$ coronary intervention; $\mathrm{MAP}=$ Mean arterial pressure; $\mathrm{ICU}=$ Intensive Care Unit

\section{The association of severe or critical hypotension with outcome}

Occurrence of any hypotension was associated with outcome. Patients without any hypotension had lower ICU- (8.9\% vs $21.6 \%, p=0.001)$ and 6 -month mortality $(22.2 \%$ vs $37.6 \%, p=0.002)$ as compared to patients with any hypotensive episode during the ICU stay, respectively. Favorable neurological outcome at six months was also more frequent, $77.2 \%$ vs $56.7 \%(p<0.001)$, in patients without any hypotension. Severe or critical hypotension was significantly associated with increased ICU $(60.7 \%$ vs $12 \%, p<0.001)$ and 6 -month mortality $(71.4 \%$ vs $27.2 \%, p<0.001)$ as well as unfavorable neurological outcome (CPC 3-5 71.4\% vs 30.7\%, p<0.001). The Kaplan-Meier graph demonstrating mortality in patients with and without severe or critical hypotension on days 1-4 is shown in Figure 1. A total of 316 patients survived the first four days. The association of any hypotension with poor neurological outcome, but not with 6-month mortality, remained significant (CPC 3-5 23.3\% with no hypotension vs $30.8 \%$ with hypotension, $p=0.014$ ) when the patients who died during the observation period (Days 1-4) were excluded from analysis. However, severe or critical hypotension was significantly associated with increased 6-month mortality $(4.4 \%$ vs $26.7 \%, p<0.001)$ in day four survivors.

\section{Comparison between 24 and 48-hour TTM-groups}

We found no difference in the incidence of severe or critical hypotension $(6.2 \%$ vs $9.7 \%$ in 24 -hour vs 48-hour treatment groups, respectively, $p=0.225)$. 


\section{Discussion}

In this post-hoc analysis of the hemodynamic data from the TTH48 study, we identified patient and resuscitation-associated factors that can be useful in predicting severe or critical hypotension from the data available on admission. This is of importance, because severe hypotension was associated with poor outcome and anticipating hemodynamic problems may provide guidance in selecting treatment modalities, i.e. hemodynamic support as well as intensified hemodynamic monitoring. Length of cooling was not associated with severe or critical hypotension in our study. We found that diabetes mellitus was strongly and independently associated with hypotensive complications. To our knowledge, our result of the strong association of hypotension and diabetes as a comorbidity is novel and has not been reported before. Previously, diabetes has been associated with decreased survival from OHCA by others $[7,8]$, but the role of hypotension was not reported. Contrary to our result, two recent studies found no difference in the frequency of diabetes in OHCA patients with high and low vasopressor dose during the post-resuscitation care, but their inclusion criteria and treatment protocols were different from that in our study. They included patients with non-shockable rhythms and the treatment protocol did not include prolonged TTM. $[9,10]$ Concerning ROSC, an association between ROSC delay and subsequent hypotension is pathophysiologically plausible due to prolonged global ischemia and our results are in concordance with results from a previous study [9]. Hemodynamic instability is a common feature of the post-cardiac arrest syndrome, the severity of which is tightly associated with time to ROSC. The pathophysiological background includes both the underlying cardiac pathology, hypoxic-ischaemic and reperfusion injury to the organ systems as well as a systemic inflammatory response, clinically manifesting as myocardial dysfunction and impaired vasoregulation and tissue oxygen delivery. [11]We also found that increasing values of MAP on admission were associated with decreasing risk for hypotension. Older patients have also been 
reported to need higher doses of vasopressors [9], but in our study age was not independently associated with severe hypotension. However, the median age of the patients with severe or critical hypotension trended to be higher [61 (53-69) vs $69(55-72), p=0.066]$ and it is possible that age would have become significantly different had the groups been larger. Indeed, in a study by Bro-Jeppesen including 920 patients, the patients with higher need for vasopressors were older than those with lower need for vasopressors and age was an independent predictor for high need for vasopressors. Their study was slightly different from ours, because they included patients also from non-shockable rhythms. They had no upper age limit in their inclusion criteria, whereas our study had an upper age limit of 80 years, excluding the extremes of ages. The mean age in our study population was somewhat lower than in their study. [9]In the study by Russo et al, hypotension was also associated with female gender, whereas we did not detect a gender-associated risk. [5] We also found that non-shockable rhythm increased the risk of subsequent severe hypotensive complications. This is likely explained with previous findings that compared to shockable rhythms, non-shockable rhythms are often associated with severe underlying illness with high Acute Physiology and Chronic Health Evaluation score 2 and more often non-cardiac causes for the arrest [12].

Our data do not elucidate whether TTM temperature would be associated with risk of severe or critical hypotension. Bro-Jeppesen et al. found that the prevalence of the highest score (4) in the cardiovascular component of the Sequential Organ Failure Assessment score (CV-SOFA) 4 was more prevalent in patients receiving $\mathrm{TTM}$ at $33^{\circ} \mathrm{C}$ compared to $\mathrm{TTM}$ at $36^{\circ} \mathrm{C}$, suggesting more severe hypotension in the patients receiving $\mathrm{TTM}$ at $33^{\circ} \mathrm{C}$. [9] They also found that more patients in the TTM at $33^{\circ} \mathrm{C}$ needed vasopressors compared to the TTM at $36^{\circ} \mathrm{C}$ group. [9] A smaller, nonrandomised observational study by Roberts et al., found no major difference in vasopressor index or MAP between patients receiving therapeutic hypothermia and normothermia [13]. In our 
population, with no difference in temperature, but a different length of $\operatorname{TTM}$ at $33^{\circ} \mathrm{C}$, more patients had some degree of hypotension in the 48-hour TTM-group [3]. There was, however, no statistically significant difference in the incidence of severe or critical hypotension, but the number of patients was too small for detecting significance. Interestingly, mild therapeutic hypothermia has been reported to reduce the circulatory instability and need of vasopressors in patients with cardiogenic shock [14] as well as the need for inotropes in post-cardiac arrest myocardial dysfunction [15]. On the other hand, a sub-study of a randomized controlled study, including OHCA patients with mild shock on admission observed a trend to higher mortality in the patients treated at $33^{\circ} \mathrm{C}$ compared to those treated at $36^{\circ} \mathrm{C}$. [16]

The incidence of hypotension in our study was comparable to other studies [1] that have also excluded patients with cardiogenic shock and severe hypotension on admission. Although present in more than $50 \%$ of patients, hypotension was mostly mild and was often controlled with single vasopressor treatment. Despite simple to treat, occurrence of even mild hypotension was associated with impaired long-term survival and poor neurological recovery. The association with post-cardiac arrest hypotension and lower survival and impaired neurologic recovery have been observed by others $[4,5,17,18]$. However, the definition of hypotension is highly variable in the literature and in many studies the role of vasopressors is not fully accounted for. [18] The protocol of the TTH-48 study defined only a lower limit of target MAP range and the choice of vasopressor use was left to the clinicians. It follows, that there may have been some differences between sites in the level of MAP that has triggered vasopressor use and a more liberal approach allowing MAP 60-65 mmHg may have been used in some centers. In our study, randomization was possible up to 24 hours from admission, which may have allowed exclusion of patients proceeding to shock within 24 hours and selected a population of OHCA patients less 
prone to develop hypotensive complications. This may partly explain the relatively low proportion of hypotension in our study.

Hypotension can be detrimental because of the loss of autoregulation in the cerebral blood flow, but the optimal blood pressure for preserving adequate blood flow is currently not known. [19, 20] Recent evidence, however, suggests that higher MAP may reduce the myocardial damage after acute ST-elevation infarction as well as neurological damage. [21, 22]

Strengths of this study include a relatively large, well characterized patient population and a prospective multi-center data collection.

There are limitations in our study that need to be addressed. First, the occurrence of hypotension was recorded on a daily basis. Second, we did not measure the total daily duration of hypotension, which could have an association with outcome measures. Third, we did not characterize in detail the cardiac pathology according to the etiology of cardiac arrest. It is possible that myocardial dysfunction was an unaccounted confounder. Fourth, we did not use the cardiovascular SOFA score in defining hypotension, which may slightly complicate comparison of our results with other recent studies. Last, the fact that hypotension often precedes death in the ICU will inevitably confound the results.

\section{Conclusions}

We found that $8 \%$ of unconscious OHCA patients undergoing TTM and post cardiac arrest care experienced severe or critical hypotension, irrespective of the duration of cooling. Diabetes, nonshockable rhythm, prolonged time to ROSC and low admission MAP were predictors of severe or 
critical hypotension. Severe or critical hypotension was associated with increased mortality and poor neurological outcome.

\section{Declarations}

\section{Ethics approval and consent to participate}

Ethical approval for the original study was obtained at each participating center. Patients were randomized after informed consent from the next of kin and patients who regained consciousness were asked for their own consent after the study, whenever possible.

\section{Availability of data and material}

The data and material are currently not publicly available. The datasets used and/or analyzed during the current study are available from the corresponding author on reasonable request.

\section{Competing interests}

Fabio Taccone reports lecture fees from BD and Zoll. Markus Skrifvars reports Speakers fees from BARD Medical (Ireland). Other authors report no competing interests.

\section{Funding}

Funding: This work was supported by Finska Läkaresällskapet and Stiftelsen Dorothea Olivia, Karl Walter och Jarl Walter Perkléns minne. 


\section{Authors' contributions}

J.H. participated in collecting the data, analyzed the data and drafted the manuscript. M.S. and H.K. conceived the idea of the study, participated in collecting the data, read and critically commented the manuscript. A.G., E.S., C.S., T.L., C.D., F.T., A.J., B.S.R., J.K., participated in data collection, read and critically commented the manuscript. All authors read and approved the final manuscript.

\section{Acknowledgements}

We thank all staff as well as patients and relatives who contributed to the TTH48 trial.

\section{References}

1) The hypothermia after cardiac arrest study group. Mild therapeutic hypothermia to improve the neurological outcome after cardiac arrest. NEJM 2002; 346:549-56. https://doi.org / 10.1056/NEJMoa012689 
2) Nielsen N, Wetterslev J, Cronberg T, Erlinge D, Gasche Y, Hassager C, et al. Targeted temperature management at $33^{\circ} \mathrm{C}$ versus $36^{\circ} \mathrm{C}$ after cardiac arrest. NEJM 2013; 369:2197206. https//doi.org/ 10.1056/NEJMoa1310519

3) Hans Kirkegaard, Eldar Soreide, Inge de Haas, Ville Pettila, Fabio Silvio Taccone, Urmet Arus, et al. Targeted temperature management for 48 vs 24 hours and neurologic outcome after out-of-hospital cardiac arrest. A randomized clinical trial. JAMA 2017; 318 :341-50. https//doi.org/ 10.1001/jama.2017.8978

4) Laurikkala J, Wilkman E, Pettilä V, Kurola J, Reinikainen M, Hoppu S, et al. Mean arterial pressure and vasopressor load after out-of-hospital cardiac arrest: Associations with oneyear neurologic outcome. Resuscitation 2016; 105:116-22. https//doi.org/ 10.1016/j.resuscitation.2016.05.026

5) Russo J, James T, Hibbert B, Yousef A, Osborne C, Wells G, et al. Impact of mean arterial pressure on clinical outcomes in comatose survivors of out-of-hospital cardiac arrest: Insights from the University of Ottawa Heart Institute Regional Cardiac Arrest Registry (CAPITAL-CARe). Resuscitation 2017; 113:27-32. https//doi.org/

10.1016/j.resuscitation.2017.01.007

6) Kirkegaard H, Rasmussen BS, de Haas I, Nielsen JF, Ikjaer S, Kaltoft A, et al. Timedifferentiated target temperature management after out-of-hospital cardiac arrest: a multicenter, randomized, parallel-group, assessor-blinded clinical trial (the TTH48 trial): study protocol for a randomized controlled trial. Trials 2016; 17: 228. https//doi.org/ $10.1186 / \mathrm{s} 13063-016-1338-9$

7) Larsson M, Thorén A, Herlitz J. A history of diabetes is associated with an adverse outcome among patients admitted to hospital alive after cardiac arrest. Resuscitation 2005; 66: $303-$ 7. 
8) Hirlekar G, Jonsson M, Karlsson T, Hollenberg J, Albertsson P, Herlitz J. Comorbidity and survival in out-of-hospital cardiac arrest. Resuscitation 2018; 133:118-23. https//doi.org/ 10.1016/j.resuscitation.2018.10.006.

9) Bro-Jeppesen J, Annborn M, Hassager C, Wise M, Pelosi P, Nielsen N, et al. Hemodynamics and vasopressor support during targeted temperature management ad $33^{\circ}$ versus $36^{\circ}$ after out-of-hospital cardiac arrest: a post hoc study of the targeted temperature management trial. Crit Care Med 2015; 43: 318-27. https//doi.org/ 10.1097/CCM.0000000000000691.

10) Bro-Jeppesen J, Kjaergaard J, Søholm H, Wanscher M, Lippert FK, Møller JE, et al.: Hemodynamics and vasopressor support in therapeutic hypothermia after cardiac arrest: Prognostic implications. Resuscitation 2014; 85: 664-70

11) Nolan JP, Neumar RP, Adrie C, Aibiki M, Berg RA, Böttiger BW, et al. Post-cardiac arrest syndrome: Epidemiology, pathophysiology, treatment, and prognostication. A Scientific Statement from the International Liaison Committee an Resuscitation; the American Heart Association Emergency Cardiovascular Care Committee; the Council of Cardiovascular Surgery and Anesthesia; the Council of Cardiopulmonary, Perioperative, and Critical Care; the Council on Clinical Cardiology; the Council on Stroke. resuscitation 2008;79:350-79

12) Vaahersalo J, Hiltunen $P$, Tiainen M, Oksanen $T$, Kaukonen K-M, Kurola J, et al. Therapeutic hypothermia after out-of-hospital cardiac arrest in Finnish intensive care units: the 
FINNRESUSCI study. Intensive Care Med 2013; 39: 826-37. https//doi.org/ 10.1007/s00134013-2868-1.

13) Roberts B, Kilgannon H, Chansky M, Jones A, Mittal N, Milcarek B, et al. Therapeutic hypothermia and vasopressor dependency after cardiac arrest. Resuscitation 2013; 84:3316. https//doi.org/ 10.1016/j.resuscitation.2012.07.029

14) Zobel C, Adler C, Kranz A, Seck C, Pfister R, Mellmich M, et al. Mild therapeutic hypothermia in cardiogenic shock syndrome. Crit Care Med 2012; 40: 1715-23. https//doi.org/ 10.1097/CCM.0b013e318246b820.

15) Jacobshagen C, Pelster T, Pax A, Horn W, Schmidt-Schweda S, Unsöld B, et al. Effects of mild therapeutic hypothermia on hemodynamics in cardiac arrest survivors and isolated failing human myocardium. Clin Res Cardiol 2010; 99:267-76. https//doi.org/ 10.1007/s00392-010-0113-2.

16) Annborn M, Bro-Jeppesen J, Nielsen N, Ullén S, Kjaergaad J, Hassager $C$, et al.; The association of targeted temperaturemanagement at 33 and $36^{\circ} \mathrm{C}$ with outcome in patients with moderate shock on admission after out-of-hospital cardiac arrest: a posthoc analysis of the Targeted Temperature Management trial- Intensive Care Med 2014; 40:1210-19

17) Kilgannon JH, Roberts BW, Reihl LR, Chansky ME, Jones AE, Dellinger $P$, et al. Early arterial hypotension is common in the post-cardiac arrest syndrome and associated with increased 
in-hospital mortality. Resuscitation 2008; 79:410-16. https//doi.org/

10.1016/j.resuscitation.2008.07.019.

18) Bhate TD, McDonald B, Sekhon MS, Griesdale DE. Association between blood pressure and outcomes in patients after cardiac arrest: A systematic review. Resuscitation 2013; 97:1-6. https//doi.org/ 10.1016/j.resuscitation.2015.08.023

19) Nolan JP, Neumar RW, Adrie C, Aibiki M, Berg RA, Böttiger BW, et al. Post-cardiac arrest syndrome: epidemiology, pathophysiology, treatment, and prognostication. A Scientific Statement from the international Liaison Committee on Resuscitation; the American Heart Association Emergency Cardiovascular Care Committee; the Council on Cardiovascular Surgery and Anesthesia. Resuscitation 2008; 79:350-79. https//doi.org/ 10.1016/j.resuscitation.2008.09.017.

20) Jakkula $P$, Pettilä V, Skrifvars MB, Hästbacka J, Loisa P, Tiainen $M$, et al. Targeting lownormal or high-normal mean arterial pressure after cardiac arrest and resuscitation: a randomised pilot trial. Intensive Care Med. 2018; 44:2091-2101. https//doi.org/ 10.1007/s00134-018-5446-8.

21) Ameloot K, Jakkula P, Hästbacka J, Reinikainen $M$, Pettilä V, Loisa $P$, et al.: Optimum Blood Pressure in Patients With Shock After Acute Myocardial Infarction and Cardiac Arrest. J Am Coll Cardiol 2020; 76:812-24 
22) Wihersaari L, Ashton NJ, Reinikainen $M$, Jakkula P, Pettilä V, Hästbacka J, et al. :

Neurofilament light as an outcome predictor after cardiac arrest: a post hoc analysis of the COMACARE trial. Intensive Care Med 2020: https://doi.org/10.1007/s00134-020-

$\underline{06218-9}$ 


\section{Figure legends}

Figure 1 Kaplan-Meier curve demonstrating different mortality after OHCA in patients with and without severe or critical hypotension during the first four post-arrest days. 
